УДК 519.812 .3

А. А. Стенин, В. П. Пасько, Е. Ю. Мелкумян, В. А. Лемешко

\title{
ФОРМИРОВАНИЕ ОПТИМАЛЬНОЙ ВАРИАТИВНОЙ ЧАСТИ УЧЕБНОГО ПЛАНА СПЕЦИАЛЬНОСТИ
}

Аннотация: В данной статье предлагается подход к формированию и оптимизации качественного перечня дисциплин вариативной части учебного плана специальности, основанный на методах экспертных оценок. При этом нет ограничений на количество экспертов, показателей качества и исследуемых дисциплин. Использование системы Интернет-опроса позволяет подключить широкий круг специалистов-экспертов для повышения доверительной вероятности оптимальности полученных результатов.

Ключевые слова: оптимизация, учебный план, Интернет-опрос, множество Парето, экспертные оценки.

\section{Введение}

Основной целью системы высшего образования является профессиональная подготовка специалистов высшей квалификации в соответствии с социальным заказом. Поэтому, именно профессиональная деятельность специалистов задает и определяет цели изучения всех учебных дисциплин, а значит и содержание, и структуру, и формы соответствующей учебной деятельности студентов, готовящихся к будущей профессиональной работе. Вот почему особое значение приобретают сейчас исследования, направленные на изучение профессиональной деятельности и разработку «портрета» специалиста того или иного профиля. Практическим результатом таких исследований явилось создание квалификационных характеристик специалистов с высшим образованием. В характеристиках дается описание основных видов деятельности специалиста, его функциональных обязанностей, требований к его подготовке. Составление квалификационных характеристик является важным этапом в решении проблемы формулировки целей подготовки специалиста и определения содержания его профессиональной деятельности.

В этом контексте большое значение приобретает формирование вариативной части учебного плана специальности. Вариативная часть дает возможность расширения и (или) углубления знаний, умений и навыков, определяемых содержанием базовых дисциплин, позволяет обучающемуся получить углубленные знания и навыки для успешной профессиональной деятельности и(или) для продолжения профессионального образования в магистратуре. Дисциплины вариативной части учебного плана специальности должны отвечать целому ряду показателей, таких как: важность дисциплины в системе подготовки специалистов данного профиля; содержательность материала дисциплины (научный уровень, глубина изложения); методический уровень изложения (логика размещения материала, ясность изложения) и другие.

() А. А. Стенин, В. П. Пасько, Е. Ю. Мелкумян, В. А. Лемешко 
Наиболее предпочтительным для формирования и оптимизации такого плана является метод экспертных оценок [1]. В этом случае, оптимальный по качественному составу дисциплин учебный план будет результатом коллективного труда экспертов как в области будущей сферы деятельности студентов данной специальности, так и специалистов высшей школы, обладающих высокой компетентностью в отношении выбранных показателей качества учебных дисциплин.

Поскольку ВУЗы, где есть данная специальность, и специалисты-эксперты данного профиля профессиональной деятельности разбросаны по всей Украине и не всегда есть возможность собрать их в одном месте, предлагается использовать систему Интернет-опроса для сбора необходимых экспертных оценок с последующей их обработкой предлагаемым ниже методом

\section{Постановка задачи}

На базе системы Интернет-опроса занесем оценки экспертов в таблицу бальных рангов учебных дисциплин (табл. 1). Здесь $r_{i j}^{k}$ - ранг (оценка) $i$-ой дисциплины $(i=1, N) j$-м экспертом $(i=1, N)$ по $k$-му показателю качества $(k=1, Q)$. Ранг представляет собой натуральное число в принятой для оценки шкале бальности.

Таблица 1. Оценки экспертов

\begin{tabular}{|c|c|c|c|c|c|c|c|c|c|}
\hline $\begin{array}{c}\text { Показатель } \\
\text { качества }\end{array}$ & \multicolumn{3}{|c|}{$J_{1}$} & $\cdots$ & \multicolumn{4}{c|}{$J_{Q}$} \\
\hline $\begin{array}{c}\text { дисциплина } \\
\text { эксперт }\end{array}$ & $k_{1}$ & $\cdots$ & $\ldots$ & $k_{N}$ & $\ldots$ & $k_{1}$ & $k_{2}$ & $\ldots$ & $k_{N}$ \\
\hline$Э_{1}$ & $r_{11}^{1}$ & $\cdots$ & $\ldots$ & $r_{1 N}^{1}$ & $\ldots$ & $r_{11}^{Q}$ & $r_{12}^{Q}$ & $\ldots$ & $r_{1 N}^{Q}$ \\
\hline$Э_{2}$ & $r_{21}^{1}$ & $\cdots$ & $\ldots$ & $r_{2 N}^{1}$ & $\ldots$ & $r_{21}^{Q}$ & $r_{22}^{Q}$ & $\ldots$ & $r_{2 N}^{Q}$ \\
\hline$\ldots$ & $\ldots$ & $\ldots$ & $\ldots$ & $\ldots$ & $\ldots$ & $\ldots$ & $\ldots$ & $\ldots$ & $\ldots$ \\
\hline$Э_{M}$ & $r_{M 1}^{1}$ & $\cdots$ & $\ldots$ & $r_{M N}^{1}$ & $\ldots$ & $r_{M 1}^{Q}$ & $r_{M 2}^{Q}$ & $\ldots$ & $r_{M N}^{Q}$ \\
\hline Сумма рангов & $\sum_{1}^{1}$ & $\ldots$ & $\ldots$ & $\sum_{N}^{1}$ & $\ldots$ & $\sum_{1}^{Q}$ & $\sum_{2}^{Q}$ & $\ldots$ & $\sum_{N}^{Q}$ \\
\hline Средний ранг & $r_{1}^{-1}$ & $\ldots$ & $\ldots$ & $r_{N}^{-1}$ & $\ldots$ & $r_{1}^{-Q}$ & $r_{2}^{-Q}$ & $\ldots$ & $r_{N}^{-Q}$ \\
\hline
\end{tabular}

Сумма рангов и средний ранг определяются соответственно по формулам:

$$
\begin{aligned}
\sum_{N}^{1} & =\sum_{j=1}^{M} r_{i j}^{k}, \\
r_{i}^{-k} & =\frac{1}{M} \sum_{i}^{k} .
\end{aligned}
$$

Не теряя общности результата, предположим, что есть 7 учебных дисциплин, которые в результате ранжирования оценок экспертов по показателям содержательности материала $\left(J_{1}\right)$ и методического уровня изложения $\left(J_{2}\right)$ сформировали следующую систему неравенств относительно среднего ранга дисциплины:

$$
\begin{aligned}
& \text { для } J_{1}: k_{6}>k_{1}>k_{2}>k_{3}>k_{7}>k_{5}>k_{4}, \\
& \text { для } J_{2}: k_{6}>k_{2}>k_{1}>k_{7}>k_{3}>k_{5}>k_{4} .
\end{aligned}
$$


Смысл неравенств (3) и (4) заключается в парных предпочтениях одного курса по отношению к другому в рамках выбранного показателя качества.

Известно, корректность полученных при обработке экспертных оценок зависит от согласованности группы экспертов. Для оценки согласованности экспертов вычисляем дисперсионный коэффициент конкордации по выбранным показателям качества [2]:

$$
W^{k}=\frac{12 S^{k}}{M^{2}\left(N^{3}-N\right)-M \sum_{j=1}^{M} T_{j}^{k}},
$$

где

$$
S^{k}=\sum_{i=1}^{N}\left(\sum_{j=1}^{M} r_{i j}^{k}-r_{0}^{k}\right)
$$

$r_{0}^{k}$ - усредненная ранговая оценка по $k$-му показателю, т.е.

$$
r_{0}^{k}=\frac{1}{N} \sum r_{i}^{-k}
$$

$T_{j}^{k}$ - показатель связанных рангов в ранжировке $j$-м экспертом, который определяется как:

$$
T_{j}^{k}=\sum_{p=1}^{H_{j}}\left(h_{p}^{3}-h_{p}\right)
$$

где: $H_{j}$ - число групп равных рангов у $j$-го эксперта, $h_{p}-$ число равных рангов в $p$-й группе связанных рангов при ранжировании $j$-м экспертом.

При $\bar{W}^{k} \geq 0,7$ считается, что группа экспертов работает согласовано. В противном случае необходимо переформировать группу экспертов.

Кроме того, целесообразно различать экспертов по степени компетентности и значимости их мнений, т.е. необходимо провести ранжирование самих экспертов. При ранжировании каждому из них присваивается ранг значимости от 1 (наиболее влиятельный эксперт) до $M$ (наименее влиятельный эксперт). Мнение каждого эксперта учитывается в расчетах с коэффициентом, пропорциональным $1 / m_{j}$, где $m_{j}$ - ранг $j$-го эксперта.

Для оценки взаимосвязи ранжированных дисциплин учебных курсов вычисляем коэффициент ранговой корреляции Спирмена[3]:

$$
\rho=1-\frac{6 S}{N^{3}-N} .
$$

Если значение $\rho$ приближается к единице, то это характеризует линейную в среднем зависимость между ранжировками по рассматриваемым показателям.

Для рассматриваемых в нашем случае двух показателей это говорит о том, что дисциплины, получившие высокую оценку по содержательности, имеют высокую оценку по методическому уровню. 
Далее проводим упорядочение дисциплин по двум показателям качества, выделяя из неравенств (3) и (4) последовательно доминирующие подмножества (множества Парето). Для наглядности дадим графическую интерпретацию указанного подхода. Для соотношений (3) и (4) на рис. 1 учебные курсы представлены согласно табл.1. подмножествами, с центральной точкой, координаты которой определяются местом данного курса среди рассматриваемых курсов, согласно его среднего ранга.

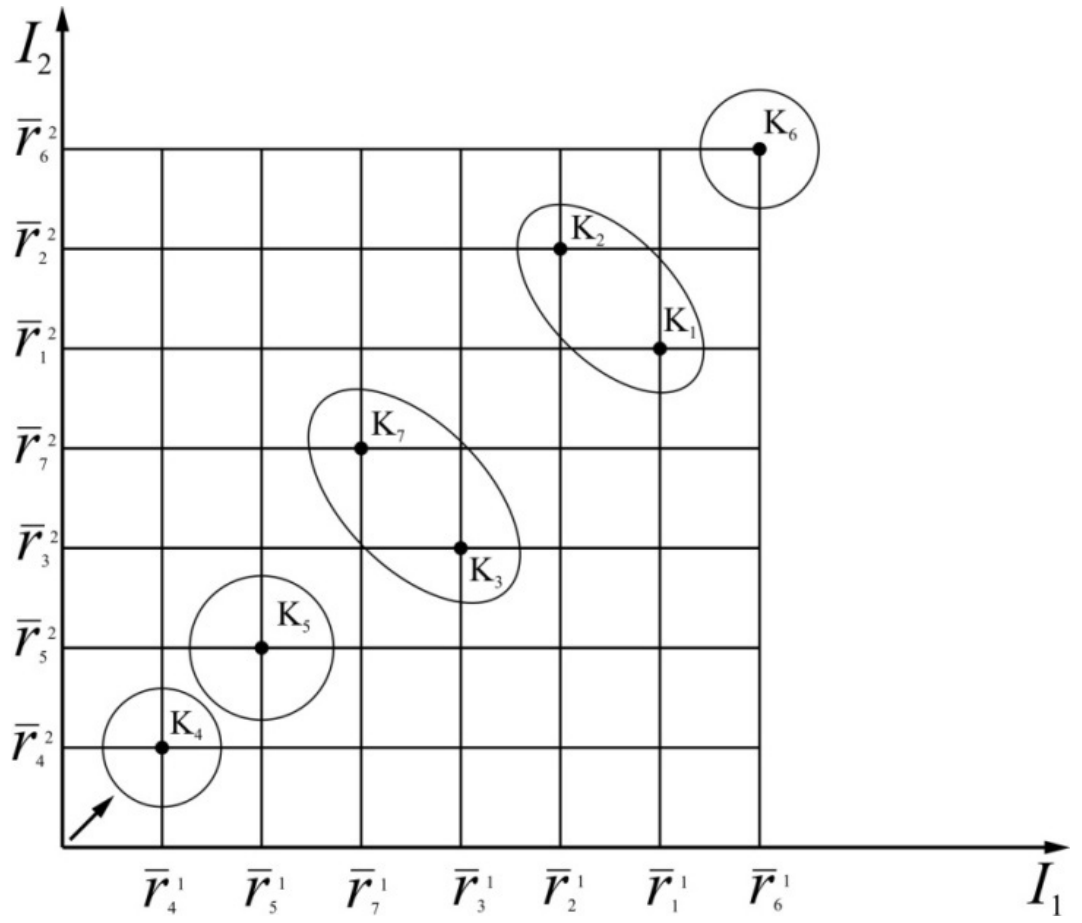

Рис. 1. - Графическая интерпретация ранговой корреляции учебных курсов

Будем считать, что показатели качества $J_{1}$ и $J_{2}$ равноценны, т.е. суммарный показатель качества определяется как:

$$
J=J_{1}+J_{2} .
$$

В этом случае вектор изменения показателя качества $J$ проходит под углом $45^{\circ}$ (рис. 1). Тогда, как видно из рис. 1, множество Парето для всей совокупности дисциплин включает только один курс $k_{6}$. Исключая курс $k_{6}$ из рассмотрения, вновь выделяем множество Парето, в которое входят два курса $k_{1}$ и $k_{2}$. Каждый из этих курсов эффективнее любого другого по обоим показателям, но между собой они несравнимы, поскольку по $J_{1} k_{1}>k_{2}$, а по $J_{2} k_{2}>k_{1}$. Продолжая эту процедуру получим следующую цепочку предпочтений учебных дисциплин:

$$
K_{6}>\left(k_{1} k_{2}\right)>\left(k_{3} k_{7}\right)>k_{5}>k_{4} .
$$

Отсюда, если, например, нам необходимо из 7 учебных курсов выбрать 5, курсы $k_{5}$ и $k_{4}$ исключаются из рассмотрения. Если же необходимо выбрать 4 курса, то помимо исключения курсов $k_{5}$ и $k_{4}$, необходимо исключить один из курсов $k_{3}$ или $k_{7}$, что можно сделать введением дополнительного показателя. Следует 
отметить, что при данном подходе при большом количестве количество показателей и дисциплин задача формирования оптимальной вариативной части учебного плана специальности существенно усложняется, что связано с анализом гиперпространства критериев качества.

В идеальном случае многокритериальной задачи можно вести поиск такого решения, которое принадлежит пересечению множеств оптимальных решений всех однокритериальных задач. Однако такое пересечение обычно оказывается пустым множеством, поэтому приходится рассматривать так называемое переговорное множество эффективных решений (оптимальных по Парето). Критерий оптимальности итальянского экономиста В. Парето применяется при решении таких задач, когда оптимизация означает улучшение одних показателей при условии, что другие не ухудшаются.

Можно выделить следующие основные методы решения задач многокритериальной оптимизации:

1. оптимизация одного, признанного наиболее важным критерия, остальные критерии при этом играют роль дополнительных ограничений;

2. сведение многих критериев к одному введением экспертных весовых коэффициентов для каждого из критериев таким образом, что более важный критерий получает более высокий вес;

3. упорядочение заданного множества критериев и последовательная оптимизация по каждому из них (этот подход лежит в основе метода последовательных уступок) [4].

Наиболее распространенным из этих методов является второй метод, использующий линейную свертку критериев на каждом уровне иерархии. В нашем случае можно выделить два уровня иерархии. На нижнем уровне формируется на основе табл. 1 критерий оценки экспертами $i$-ой дисциплины по $k$-му критерию. Обозначим его как $E_{i k}$. Из табл. 1 видно, что $E_{i k}=r_{i}^{-k}$. Тогда критерий верхнего уровня для оценки $i$-ой дисциплины по совокупности критериев нижнего уровня будет иметь вид:

$$
J_{i}=\sum_{k=1}^{Q} \lambda_{i k} E_{i k}
$$

где $\lambda_{i k}-$ весовые коэффициенты важности $k$-го критерия при оценке $i$-ой дисциплины, которые из табл. 1 определяются как:

$$
\lambda_{i k}=\sum_{k=1}^{Q} r_{i}^{-k} / \sum_{i=1}^{N} \sum_{k=1}^{Q} r_{i}^{-k},
$$

причем

$$
\sum_{k=1}^{Q} \lambda_{i k}=1, \lambda_{i k} \geq 0
$$

Учитывая целочисленное ранжирование критериев, что приводит к болышому диапазону разброса оценок, для более точной оценки предлагается использовать 
свертку относительных значений критериев $i$-ых дисциплин относительно их максимальных и минимальных значений [3,5]. Способ свертки состоит в решении задачи минимизации линейной комбинации с неотрицательными весовыми коэффициентами $\lambda_{i k}$, обозначающими важность $k$-го критерия, и удовлетворяющие условию (14):

$$
J_{i}=\sum_{k=1}^{Q} \lambda_{i k}\left(E_{i k \max }-E_{i k}\right) /\left(E_{i k \max }-E_{i k \min }\right) .
$$

Далее, согласно полученным из (12) значениям критериев верхнего уровня, ранжируем в порядке убывания список дисциплин и отбираем заданное количество дисциплин в вариативную часть учебного плана специальности.

После формирования оптимальной по качественному составу дисциплин вариативной части учебного плана специальности следующей важной задачей повышения уровня подготовки специалистов является формирование моделей и целевых показателей учебного процесса [6].

\section{Заключение}

Предложенный подход к формированию и оптимизации вариативной части учебного плана специальности носит универсальный характер и может быть использован для решения подобных задач в различных сферах деятельности человека. При этом нет ограничений на количество экспертов, показателей качества и исследуемых объектов. Главная проблема состоит в многокритериальности решаемой задачи. При небольшом числе критериев она решается на основе парной доминантности критериев так, как это показано в данной статье. При большом числе критериев предлагается использовать линейную свертку. При этом использование системы Интернет-опроса позволяет подключить широкий круг специалистов-экспертов для повышения доверительной вероятности оптимальности полученных результатов.

\section{Список использованных источников}

1. Нейлор К. Как построить экспертную систему. - М.: Энергоатомиздат, 1991.- 286 с.

2. Добров Г.М. и др. Экспертные оценки в научно-техническом прогнозировании. К.: Наукова Думка, 1974. - 160 с.

3. Бешелев С.Л., Гурвич Ф. Г. Математические методы экспертных оценок. - М.: Статистика, 1980. - 263 с.

4. Подиновский В.В., Гаврилов В. М. Оптимизация по последовательно применяемым критериям. М., “Сов. радио”, 1975. - 192 с.

5. Орлов А.И. Экспертные оценки. - М.: Изд-во МГТУ им. Н.Э. Баумана. - 2009. Ч. 2 : $-2011 .-486 \mathrm{c.}$

6. Стенін О.А.,Тімошин Ю.А., Домаскіна Н.І., Шалабай І.П. Моделі і цільові показники учбового процесу в автоматизованих учбових системах. Адаптивні системи автоматичного управління// Міжвідомчий науково-технічний збірник. - Дн-ск, 2006, вип. 9(29).- стр. 140-147. 